\title{
Determining Tarsus Curvature in Relation To Direction of Gaze Using Non-contact Ultrasound Video Assistance
}

Authors

Affiliations

\section{T. Schrom , R. Amberg², F. Bast}

${ }^{1}$ Head and Neck Surgery/Plastic Surgery, Clinic for Oto-Rhino-Laryngology, Bad Saarow, HELIOS Clinics Bad Saarow, Germany

${ }^{2}$ Institute for International Media and Computing, Economics II, University of Applied Science, Berlin, Germany

${ }^{3}$ Department for Otolaryngology, AMEOS Klinikum Haldensleben, Haldensleben, Germany
Key words

- eye

- face

- ultrasound received $\quad 03.05 .2015$

accepted $\quad 31.10 .2015$

\section{Bibliography}

DOI http://dx.doi.org/

10.1055/s-0035-1565243

Published online:

January 15, 2015

Ultrasound International Open

2016; 2: E8-E12

(c) Georg Thieme Verlag KG

Stuttgart · New York

ISSN 2199-7152

\section{Correspondence}

Dr. F. Bast

Department for Otolaryngology AMEOS Klinikum Haldensleben

Kiefholzstrasse 4

39340 Haldensleben

Germany

Tel.: + 49/178/4155 269

FlorianBast@gmx.de

\section{License terms}

\section{(ब(1) $\Theta \circledast$}

\section{Abstract}

\section{$\nabla$}

Purpose: Plastic surgery on the eyelids for the purpose of aesthetic or functional correction requires precise knowledge of lid anatomy. Changes in the tarsal curvature of the upper eyelid relative to line of vision are important, particularly when a surgical correction of paralytic lagophthalmos is undertaken. We used a computer-based image-processing algorithm to establish a relationship between changes in the curvature of the tarsus relative to the line of vision.

Material and Methods: A dynamic, ultrasound examination of the upper eyelids of 100 participants (100 eyes) was performed transpalpebrally

\section{Introduction}

$\nabla$

Plastic surgery on the eyelids for the purpose of aesthetic or functional correction requires precise knowledge of lid anatomy. The average width of the palpebral fissure is ca. $34 \mathrm{~mm}$. The distance between the edges of the upper and lower lids is $10-12 \mathrm{~mm}$. The average axial length ranges from 23.5 to $24.5 \mathrm{~mm}$ with a three-layered wall; the average corneal radius is between 7.7 and $8.3 \mathrm{~mm}$. The eyelids, located in front of the globe, serve to protect the eye, in particular the sensitive cornea. While there is comprehensive research on the anatomic measurements of the anterior portions of the eye, the corneal radius, the axial length and eye socket spacing, there is little data regarding the functional anatomy of the upper eyelid [1-6].

An ultrasound examination of the eye and socket can be carried out either transpalpebrally or directly on the locally anesthetized eye. This type of examination has been used for various eye diseases [7-11]. As a dynamic examination method, ultrasound is ideally suited for detecting func- using a $7.5 \mathrm{MHz}$ scanner with the patient looking straight ahead, away from and towards the midline of the body. A computer-aided examination of the upper eyelid tarsus was then performed, followed by the calculation of the radius of curvature of the tarsus relative to the line of vision in each position.

Results: Using regression of a Taylor polynomial, the shape of the tarsus was mapped by a quadratic function, and the change in tarsal curvature relative to line of sight could be demonstrated.

Conclusion: With objective evidence of change in the tarsal curvature relative to the line of sight, this may influence the treatment of pathological changes in the upper eyelid. tional changes in the upper lid. The procedure is also complication-free and inexpensive. In a study published in 2001 it was shown that the upper lid tarsus, a plate of connective tissue, can be easily measured and depicted through ultrasound using a $7.5 \mathrm{MHz}$ linear scanner transpalpebrally and the so-called non-contact procedure. The results included not only differences in tarsus curvature among healthy individuals, but also differences in tarsus curvature depending on gaze direction [12]. This change in the curvature of the upper lid tarsus depending on the line of sight remains even in paralytic lagophthalmus despite the loss of tone in the orbicularis oculi. The preservation of "upper lid flexibility" is especially important in the surgical correction of lagophthalmus $[13,14]$. However, in the abovementioned study only extreme values were determined (gaze straight ahead, left and right). The determination and detection of the upper lid tarsus was carried out manually with the help of individual ultrasound images and therefore remain subjective and dependent on the examiner. 
The aim of this paper is to introduce a method of performing a simple, non-invasive test, the data from which may be inputted into a formula that efficiently generates specific information that may benefit and change their clinical practice and decision making in the future.

\section{Materials and Methods}

$\nabla$

Assessment and calculations were carried out in close cooperation with the Institute for International Media and Computing, Economics II, University of applied Science, Berlin. The computer-based algorithm was tested in a total of 100 subjects with healthy eyes (100 eyes measured). Upon request to the Ethics Commission in Cottbus, Brandenburg, Germany, no official approval for this study was needed. There were 57 female and 43 male subjects, with an average age of 39.2 years. All were being treated for unrelated ENT complaints in our department. For this study a Toshiba Aplio CV SSA-770 A ultrasound device was used in combination with a PLT-604AT (5-11 MHz) transducer. The examinations were conducted transpalpebrally using the non-contact procedure. For transmission, conventional ultrasound gel was used. Since anatomically no significant impedance in the upper lid area is expected (i.e., bones or air) the B-scan linear scanner accurately captures the geometry of the proportions. The ultrasound transducer was placed vertically over the closed eye ( $\bullet$ Fig. 1a), without touching it or applying pressure. During the examination neither the transducer nor the patient was bound. This approach has proven successful and has been reported in numerous published articles [12-14].

First the ultrasound was performed on the upper lid with the gaze directed straight ahead and then toward and away from the midline of the body. This step was recorded as a video sequence. In addition, computer-based investigations of the globe via Hough-Transformation of the iris with its angular deflection corresponding to changes in line of vision ( $\bullet$ Fig. $1,2,3$ ) and the upper lid tarsus ( $\bullet$ Fig. $\mathbf{2 , 4}$ ) were conducted. These investigations were undertaken simultaneously with the examination. Afterward the image and calculations of the tarsal curvature radius, depending on the angle, were presented in chronological sequence ( $\bullet$ Fig. 3 ).

\section{Analysis of video data}

In order to develop the most precise algorithm for detecting tarsus curvature, it is of the utmost importance to be able to clearly identify and mark the anatomical structures depicted on the ultrasound videos. The image in $\bullet$ Fig $\mathbf{1 a}$, b shows the left eye of one of our subjects. Because the ultrasound signal is directiondependent, only those structures positioned vertically in relation to the pulse are visible. Prominent areas such as the upper lid (marked in blue), iris (red) and fundus (yellow) are easy to see. Even parts of the lens (green) are recognizable in this image. These factors provide a good basis for a computer-based assessment of the upper lid and the curvature.

\section{Representation of the eyeball}

Since the position of the lens and tarsus depends on the eyeball, its representation constitutes an important step in the analysis. The sides of the eyeball are not visible because of the way the ultrasound works: the ultrasound signal moves straight out from the wand and therefore only detects structures positioned vertically in relation to the signal. The image of the fundus and the circular shape shown by the cornea and edge of the upper lid ( $\bullet$ Fig. 4a) were sufficient to successfully represent the eyeball by means of a Hough Transformation for circles. The Hough Transformation recognizes geometric structures (i.e., lines or circles) in a binarized image. The Hough Transformation is very robust in cases of interference or partially hidden objects [15].

\section{Representation of the lens}

After detecting the eyeball comes the lens, which is found within the circle detected by the Hough Transformation. Since the lens is primarily made up of water and therefore exhibits an acoustic impedance similar to that of hyaloid fluid, it is either badly or not at all detected in the ultrasound video. In $\bullet$ Fig. 1b, only the protein-rich outer layer of the lens is recognizable, which the amplitude of the sound pulse changes slightly. In addition to the lens, the pupil, surrounded by the iris, also gives information about gaze direction. The iris ( $\bullet$ Fig. 4b) appears in the ultrasound image as a somewhat thicker line interrupted by the pupil. If a perpendicular line is drawn from the midpoint of the eye to the iris, it traverses the pupil. Assuming that $0^{\circ}$ is straight ahead, the line of sight is then defined as a positive or negative deviation from the eye axis.

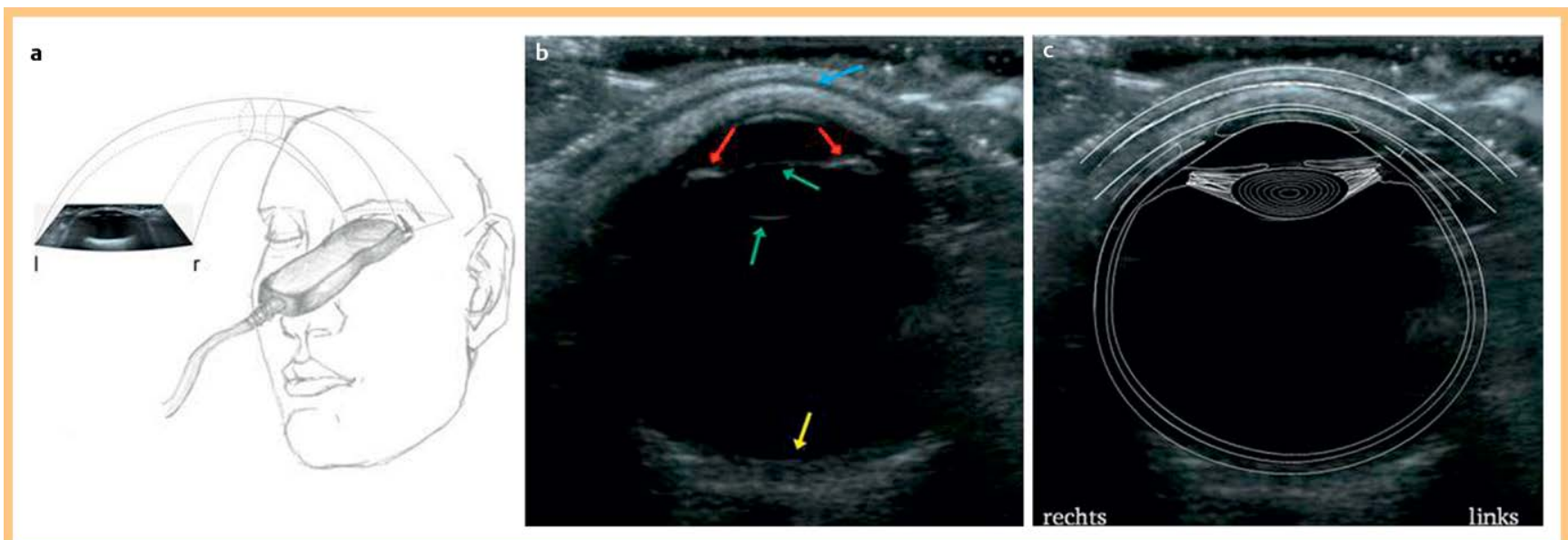

Fig. 1 a Examination configuration using ultrasound probe $\mathbf{b}$ Transpalpebral ultrasound image of a study subject showing iris (red), lens (green), fundus (yellow), upper lid (blue) $\mathbf{c}$ Identification of the anatomical components of the eye. 


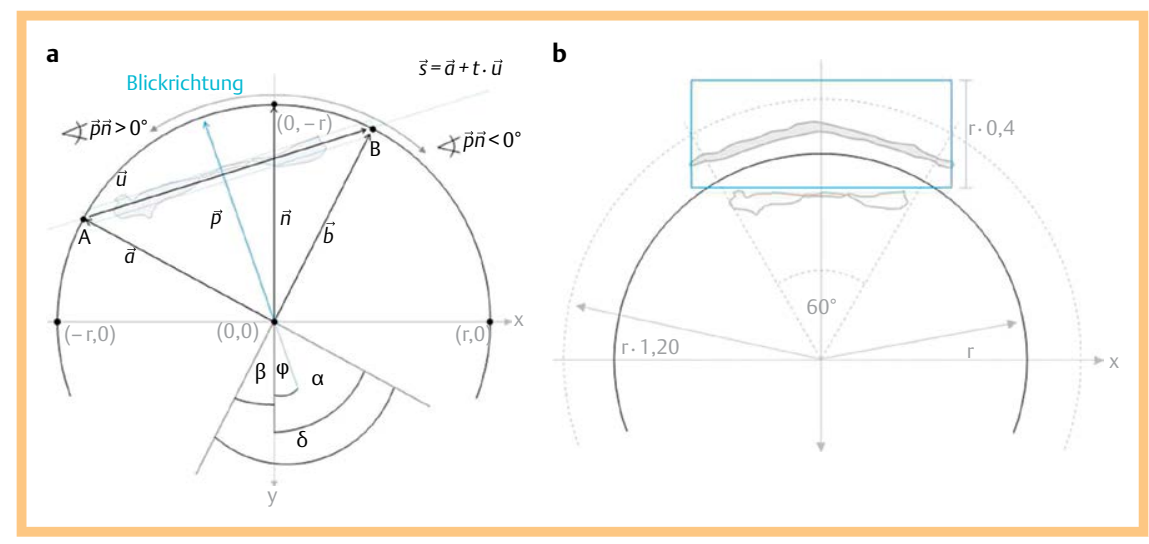

Fig. 2 Representation of the vectors and their angle relationships, necessary for a Detecting the iris and eyeball using Hough Transformation and b calculating the search field for detection of the tarsus taking measurements from straight ahead gaze transecting the pupil, $r=$ radius.
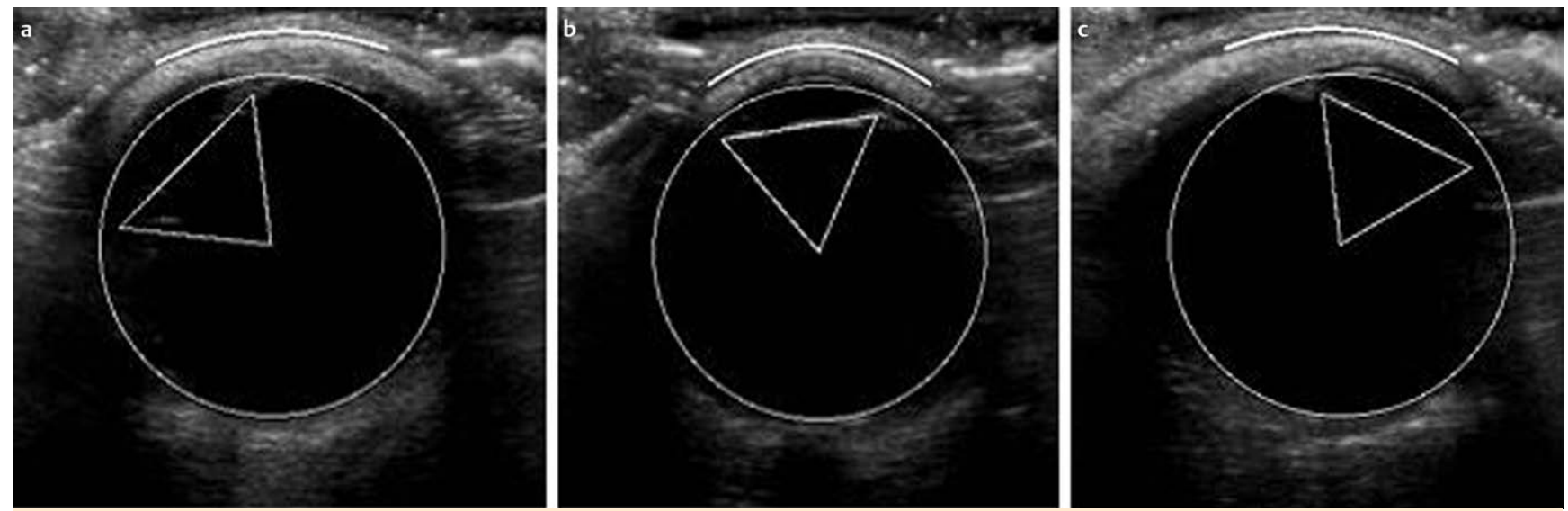

Fig. 3 Detected eyeball, iris and tarsus represented on ultrasound scan pictures in 3 distinct gaze directions: a left, $\mathbf{b}$ straight ahead, $\mathbf{c}$ right.
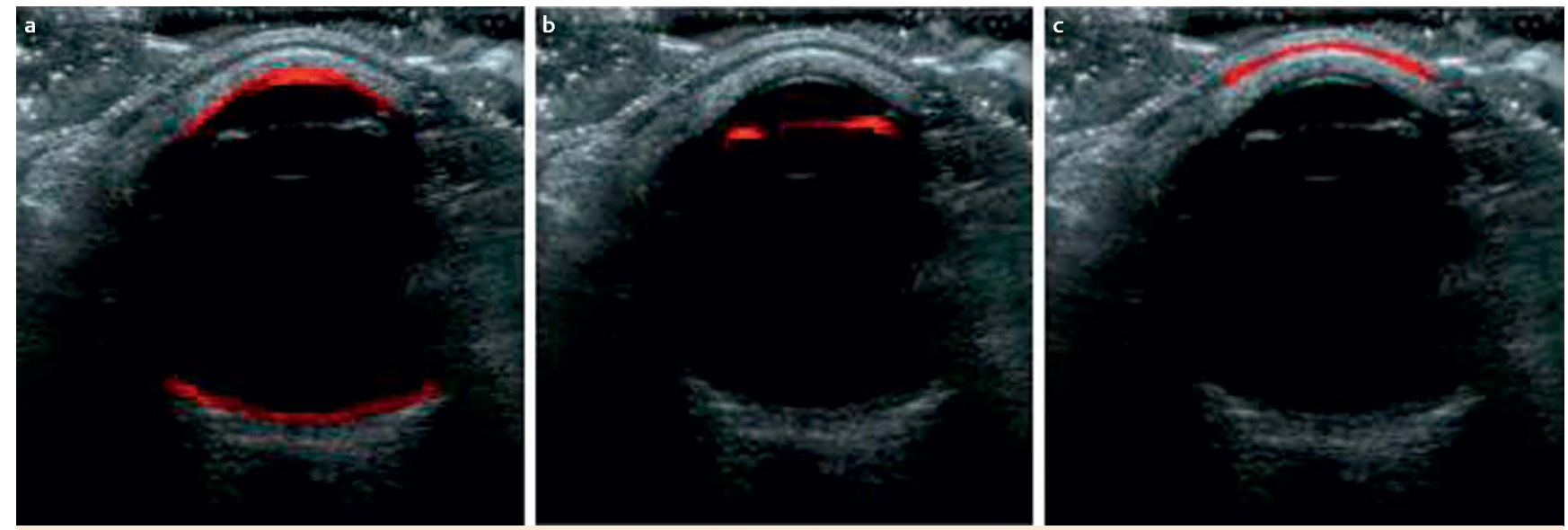

Fig. 4 Transpalpebral ultrasound picture showing areas important for the detection of the eyeball. a Cornea b Iris and $\mathbf{c}$ Tarsus section above the eyeball.

\section{Detection of the upper eyelid and tarsus}

Since it is relatively difficult to directly detect the upper lid, it is helpful to use a roundabout method. The tarsus is located within the lid, and due to its lower water content is clearly distinguished from the rest of the upper lid tissue in the ultrasound ( $\bullet$ Fig. 4c). Because of the anatomy of the upper lid, the tarsus exhibits a nearly identical curvature along the length of upper lid as the lid itself. Therefore together with the determination of the tarsus curvature, the curvature of the upper lid is also determined. Moreover, the upper lid and tarsus do not change their position with eye movement. Only the shape or curvature changes according to the line of sight. After detection of upper lid and tarsus, the determination of the curve radius of the tarsus was carried out depending on gaze direction. This was done starting from a defined point on the tarsus. The point chosen is the one located precisely over the midpoint of the eyeball when the gaze is directed straight ahead. If the eyeball circle is extended by $20 \%$ and 2 lines are drawn from the midpoint upwards each with a distance of $30^{\circ}$ left and right of the vertical axis, 2 points of intersection are produced within the extended circle. If a square is drawn over these points whose vertical edges amount to $40 \%$ of the radius, the tarsus is precisely in this square ( 0 Fig. $\mathbf{2 a}$ a b). This type of localization was developed as part of this project and is based on the anatomical substructures of the eye as well 
as on the ultrasound videos taken in the course of our work. The tarsus, a layer of connective tissue embedded in the upper lid, is distinguished in the video as a white strip. Viewed on the eyeball, the first side runs from black (hypo echoic) to white (hyper echoic), and the second from white to black.

Following successful detection of the tarsus, its shape was depicted using regression of a Taylor polynomial with a quadratic function. Taylor's Theorem is used to approximate functions around a given point with polynomials, so-called Taylor polynomials. For a simplified interpretation, the curvature at the vertex of the quadratic function was transformed into the radius of curvature of an imaginary circle drawn through the upper lid. When both parameters (line of sight and radius of curvature) have been successfully detected, the Taylor polynomial was once again used to develop the formula describing the relationship between radius of curvature of the upper lid and line of sight.

\section{Results}

$\nabla$

Upon detection of the tarsus its shape was depicted using regression of a Taylor polynomial with a quadratic function. We were able to objectively demonstrate a relationship between tarsal curvature relative to the line of sight and have developed a formula to define this relationship.

When the gaze is directed straight ahead the upper lid is extremely curved and creates a small circle, while when the gaze is directed to the left or right the upper lid curvature creates a larger circle ( $\bullet$ Fig. 3 ). With this used formula it is possible, in the current range, to demonstrate the dependence of the upper lid curvature on the line of sight ( $\bullet$ Fig. 5). Using this process, the final analysis of an ultrasound video consisting of 315 single images of conventional size, takes approximately $4 \mathrm{~min}$ - including optimization.

\section{Discussion \\ $\nabla$}

The eyelids fulfil 2 main functions. On one hand, they serve to protect the eye from all types of contact, foreign bodies, injuries and light, as well as from mechanical, chemical or other damaging and undesirable influences. Beyond their protective function, the eyelids play a significant role in the creation of facial expression. They contribute to the impression others receive as to a person's current state or personality and help communicate surprise, fear, joy, sadness or fatigue.

For aesthetic and plastic-reconstructive procedures in the area of the eyelids, knowledge of their anatomy is essential. While there are comprehensive studies on the anatomic measurement of the eye, there is little data on the functional anatomy of the upper lid [3-6].

In order to capture the functional changes of the upper lid, ultrasound is ideally suited as a dynamic examination method. It is able to demonstrate that there are not only individual differences in tarsus curvature, but that tarsus curvature changes according to the line of sight as well [12].

This change in the curvature of the upper lid tarsus with a change in the line of sight remains even in paralytic lagophthalmus, despite loss of muscle tone in the orbicularis oculi. The preservation of "upper lid flexibility" is especially important in the surgical correction of lagophthalmus $[13,14]$. However in the latter studies only the determination of extreme values (gaze direction straight ahead, left and right) were carried out. Determination and detection of the upper lid tarsus was manually performed based on individual ultrasound images, and thus remains subjective and dependent on the examiner.

For this reason an algorithm was developed capable of objectively determining line of sight and tarsus curvature using ultrasound videos. On the basis of data derived from the ultrasound videos, a formula was created which sufficiently describes the dependence of tarsus curvature on line of sight. For this purpose, the eyeball was first detected in the video, then the lens

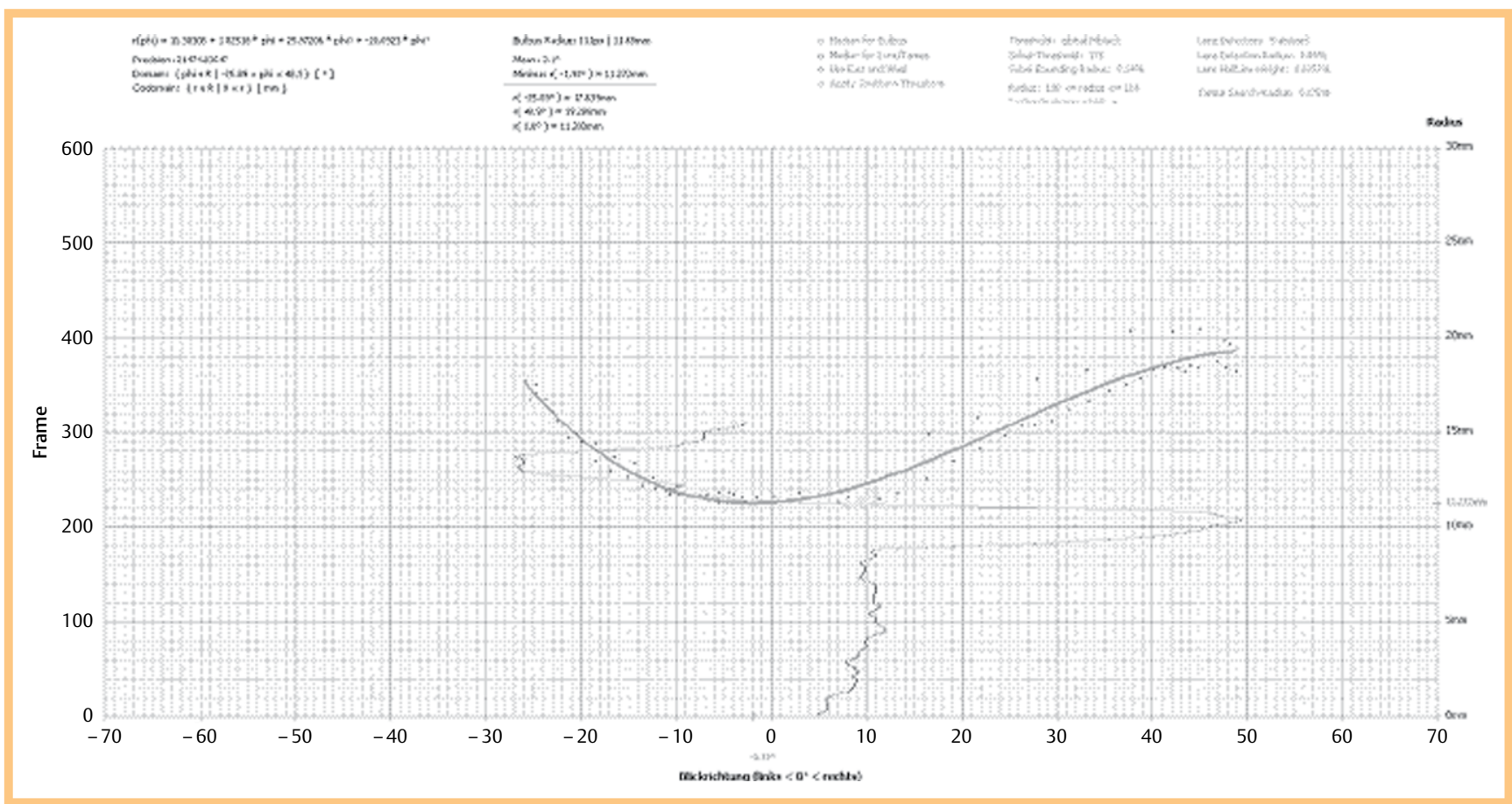

Fig. 5 Graph representing the correlation between the line of sight ( $x$-axis with 0 being straight ahead gaze), and the radius of the upper lid ( $y$-axis). 
and upper lid including the tarsus. Then the line of sight could be detected in the video. After the successful detection of the line of sight, the shape of the tarsus, located in the upper lid, was depicted in the form of a quadratic function. The precise detection of the tarsus curvature may be usful before surgical correction of deformities of the upper lid $[16,17]$, or to assist the development of flexible or customized lid implants as treatment for paralytic lagophthalmus, since inflexible standard implants do not meet the anatomical requirements $[13,18,19]$.

\section{Conclusion}

We tried to introduce a novel method to clinicians to determining the relationship of tarsus curvature to changes in line of sight based on ultrasound videos and a computer-supported algorithm. From this data a formula was created which describes the dependence of the tarsus curvature on changes in line of sight. With evidence that the tarsus curvature changes in relation to gaze direction, this data may beneficially influence the treatment of abnormalities in the upper lid.

\section{Conflict of Interest}

$\nabla$

No conflict of interest for all authors.

\section{References}

1 Rauber A, Kopsch F. Nervensystem - Sinnesorgane. In: Lehrbuch und Atlas der Anatomie des Menschen. Stuttgart: Thieme Verlag; 2003

2 Amini R, Jouzdani S, Barocas VH. Increased iris-lens contact following spontaneous blinking: mathematical modeling. J Biomech 2012; 45: 2293-2296

3 Kakizaki H, Leibovitch I, Selva D et al. Orbital septum attachment on the levator aponeurosis in Asians: in vivo and cadaver study. Ophthalmology 2009; 116: 2031-2035
4 Connell B, Brian G, Bond MJ. A case-control study of biometry in healthy and cataractous Eritrean eyes. Ophthalmic Epidemiol 1997; 4: $151-155$

5 Richard MJ, Morris C, Deen BF et al. Analysis of the anatomic changes of the aging facial skeleton using computer-assisted tomography. Ophthal Plast Reconstr Surg 2009; 25: 382-386

6 Pierro L, Brancato R, Robino $X$ et al. Axial length in patients with diabetes. Retina 1999; 19: 401-404

7 Jank S, Deibl M, Strobl $\mathrm{H}$ et al. Intrarater reliability in the ultrasound diagnosis of medial and lateral orbital wall fractures with a curved array transducer. J Oral Maxillofac Surg 2006; 64: 68-73

8 Laws F, Laws D, Wood I et al. Assessment of a new through-the-eyelid technique for ' $\mathrm{A}$ ' scan ultrasound ocular axial length measurement. Ophthalmic Physiol Opt 1998; 18: 408-414

9 Aviv RI, Miszkiel K. Orbital imaging: Part 2. Intraorbital pathology. Clin Radiol 2005; 60: 288-307

10 Twelker JD, Kirschbaum S, Zadnik K et al. Comparison of corneal versus through-the-lid A-scan ultrasound biometry. Optom Vis Sci 1997; 74: 852-858

11 Lee HS, Lew H, Yun YS. Ultrasonographic measurement of upper eyelid thickness in Korean children with epicanthus. Korean J Othalmol 2006; 20: 79-81

12 Schrom T, Grube A, Goldhahn A et al. Sonographic imaging of upper eyelid tarsal radii when changing the direction of vision. Ultraschall Med 2001; 22: 172-175

13 Schrom T, Bloching $M$, Wernecke $K$ et al. Measurement of upper eyelid implants curvature by ultrasound. Laryngoscope 2005; 115: 884-888

14 Schrom T, Wernecke K, Debelius A et al. Ultrasound of the upper eyelid to evaluate tarsal curvature in facial palsy. Klin Monbl fur Augenheilkd 2006; 223: 285-288

15 Thomae N, Plagwitz KU, Husar P et al. Hough transformation for image processing in eye tracking recording. Biomed Tech (Berl) 2002; 47 (Suppl 1): Pt 2 636-638

16 Lew H, Yu SB, Yun YS et al. Correction of epiblepharon of the upper eyelid by the buried suture technique: correlation with morphological features of the upper eyelid. Ophthalmologica 2008; 222: 100-104

17 Lee H, Park M, Lee TE et al. Surgical correction of epiblepharon using thermal cauterization of the orbital septum and lash-rotating sutures. J Craniofac Surg 2010; 21: 1069-1071

18 Schrom T, Habermann A, Wernecke K et al. Implantation of lid weights for therapy of lagophthalmos. Ophthalmologe 2005; 102: 1186-1192

19 Schrom T, Habermann A, Wernecke K et al. Lidloading and intraocular pressure. Klin Monbl Augenheilkd 2005; 222: 46-49 